\title{
Integrative Bioinformatics Analysis of iNOS/NOS2 in gastric and colorectal cancer
}

https://doi.org/10.1515/pteridines-2020-0011

received July 20, 2018; accepted August 22, 2018.

\begin{abstract}
Objective The aim of the present work was to investigate the expression of nitric oxide synthase 2 (iNOS/ NOS2) in colorectal and gastric cancers and evaluate its association with patient's prognosis by integrated
\end{abstract} bioinformatics analysis.

Methods The data for present study was obtained from the TCGA, GTEx, and STRING database. iNOS/NOS2 mRNA expression in normal tissue and colorectal, and gastric cancer tissuea were investigated through the GTEx and TCGA database. iNOS/NOS2 gene mutations and frequency were analyzed in the TCGA database using the cBioPortal online data analysis tool. The protein-protein interaction (PPI) network of iNOS/NOS2 was constructed by STRING database. Gene ontology (GO) and Kyoto Encyclopedia of Genes and Genomes (KEGG) pathway of iNOS/NOS2 and relevant proteins involved in the PPI network were enriched and demonstrated by the bubble plot. Comparison of the overall survival(OS) and disease free survival(DFS) between samples expressing high and low levels of iNOS/NOS2 was analysis based on the TCGA databases through the GEPIA online data analysis tool.

Results For colon adenocarcinoma (COAD) and rectal adenocarcinoma(READ) iNOS/NOS2 mRNA expression levels in tumor tissue were significant higher than those of corresponding normal colorectal tissue $(p<0.05)$. iNOS/NOS2 mutations were identified in both colorectal cancer and gastric cancer. Missense substitutions and synonymous substitution were the top two mutation types for colorectal and gastric cancer. The top positive and negative co-expressed genes correlated with iNOS/ NOS2 were TRIM40 $\left(\mathrm{r}_{\text {pearson }}=0.56, \mathrm{p}<0.05\right)$ and GDPD5

\footnotetext{
*Corresponding author: Suping Wu, Department of ICU, Lishui People's Hospital, Lishui 323000, Zhejiang Province, China, E-mail: 503745717@qq.com

Mingbei Lu, Department of Thyroid and Breast Surgery, Lishui People's Hospital, Lishui 323000, Zhejiang Province, China Guoxiong Cheng, Chaobo Xu, Zhengwei Chen, Department of Gastrointestinal Surgery, Lishui People's Hospital, Lishui 323000, Zhejiang Province, China
}

$\left(\mathrm{r}_{\text {pearson }}=-0.41, \mathrm{p}<0.05\right)$ in colorectal cancer respectively andCASP5 $\left(\mathrm{r}_{\text {pearson }}=0.63, \mathrm{p}<0.05\right)$ and PIAS3 $\quad\left(\mathrm{r}_{\text {pearson }}=-\right.$ $0.43, p<0.05)$ in gastric cancer. Twenty one proteins were included in the PPI network with 51 nodes and 345 edges which indicated the PPI enrichment wassignificant ( $p=1.0 e-16)$. The KEGG of the included genes were mainly enriched in metabolic pathway and Jak-STAT signaling pathway. There was a significant difference indisease free survival (DFS) between samples expressing high and low iNOS/NOS2 $(\mathrm{HR}=0.37, \mathrm{p}=0.044)$ in rectal cancer. The difference was not statistical between iNOS/NOS2 high and low expressing groups for overall survival(OS) or DFS in the colon cancer or gastric cancer( $p>0.05)$.

Conclusions iNOS/NOS2 mRNA isup-regulated in tumor tissue compared to corresponding normal tissue in colorectal and gastric cancer which implement it in the development of colorectal and gastric cancers.

Keywords: iNOS/NOS2; bioinformatics; colorectal cancer; gastric cancer; survival.

\section{Introduction}

Gastric and colorectal cancers are the most diagnosed malignant tumors of the digestive system. It was estimated 27600 new stomach and 147950 new colorectal cancer cases would be diagnosed in the year 2020 [1]. The general prognosis of gastric and colorectal cancers are poor due to advanced stages when fist diagnosis [2-4]. Although gastric and colorectal cancer are frequent and have poor prognosis, the molecular carcinogenesis for their development, cell migration and invasion are not completely understood yet. In recent years, many studies have confirmed that driving genes play an import role in the carcinogenesis and pathways [5, 6]. Several driving genes such as tp53, p16ink4a had been found played an essential role in tumor genesis [7, 8].

Nitric oxide synthase 2 (NOS2) also known as inducible nitric oxide synthase(iNOS) is an important member of NOSs family which encodes enzymes that catalyze the production of nitric oxide (NO) from L-arginine. Several studies had confirmed that iNOS/NOS2 are up-regulated in 
tumor tissues compared to corresponding normal tissues in multiple cancers such as breast cancer, colorectal cancer, esophageal cancer, gastric cancer, prostate cancer [9-11]. However, integrated bioinformatics analysis of iNOS/NOS2 in gastric and colorectal cancer considering its expression, mutational status, gene ontology, KEGG pathway and prognosis are yet to be reported.

\section{Methods}

\section{iNOS/NOS2 mRNA expression analysis}

iNOS/NOS2 mRNA expression in human normal tissue were explored using the GTEx databases. iNOS/NOS2 mRNA expression in colorectal cancer and gastric cancer were evaluated using the online data analysis tool GEPIA(http://gepia.cancer-pku.cn/detail.php) based on the TCGA database(https://portal.gdc.cancer.gov/).

\section{iNOS/NOS2 gene mutation and co-expression analysis}

iNOS/NOS2 gene mutation type and frequency were explored using the cBioPortal (http://cbioportal.org) based on the TCGA database. The mutation frequency of nonsense substitution, missense substitution, synonymous substitution, in-frame insertion, frame shift were identified and expressed using a pie chart. The single nucleotide mutation of iNOS/NOS2 were also screened using the catalogue of somatic mutation in cancer (COSMIC) (https://cancer.sanger.ac.uk/cosmic/) online data analysis tool [12]. Co-expressed genes were clustered and demonstrated by the heatmap generated from LinkedOmics database (http://www.linkedomics. org/login.php) [13]. with the genes with the top positive and negative correlation with iNOS/NOS2 were identified and statistical significance calculated using the Pearson correlations test.

\section{PPI network construction and GO and KEGG pathway enrichment}

The protein-protein interaction (PPI) network was constructed using the STRINIG database (http://string-db. org/cgi/input.pl) [14]. Biological function and pathways of iNOS/NOS2 and relevant genes were enriched using gene ontology(GO) including biological process (BP), cellular component (CC) molecular function (MF) and KEGG pathway analysis. A Bubble plot was used to demonstrated the biological functions and pathways enriched [15].

\section{Survival analysis}

Survival data of colorectal and gastric cancer patients from the TCGA database were used to analyst overall survival (OS) and disease free survival (DFS) when comparing iNOS/NOS2 high and low expression groups. The iNOS/NOS2 high expression cases were defined as the patients with iNOS/NOS2 expression more than the median expression level of the tumor tissue.

\section{Statistical analysis}

The data was analyzed based on the relevant databases or online data analysis tool. In survival analysis, the patients were divided into iNOS/NOS2 high and low expression according to the median iNOS/NOS2 mRNA expression. The overall survival(OS) and disease free survival(DFS) were demonstrated by the survival curve and comparison between high and low iNOS/NOS2 expression group was made using a log-rank test.

\section{Results}

\section{iNOS/NOS2 mRNA expression}

The iNOS/NOS2 mRNA levels of human normal tissues were heterogenous (Fig 1A). For colon adenocarcinoma (COAD) and rectal adenocarcinoma (READ) iNOS/NOS2 mRNA expression level in tumor tissues were significant higher than those of corresponding normal colorectal tissues $(\mathrm{p}<0.05)$ (Fig 1B, C). For gastric cancer, iNOS/ NOS2 mRNA was up-regulated in tumor tissue compared to corresponding normal gastric tissue, but not to a statistically significant level ( $p>0.05$ ) (Fig 1D).

\section{iNOS/NOS2 mutation analysis}

iNOS/NOS2 mutations were identified in both colorectal cancer and gastric cancer. The mutation percentage was calculated by considering the number of cases with mutation against the number of cases studied. Missense substitutions, synonymous substitutions and nonsense 


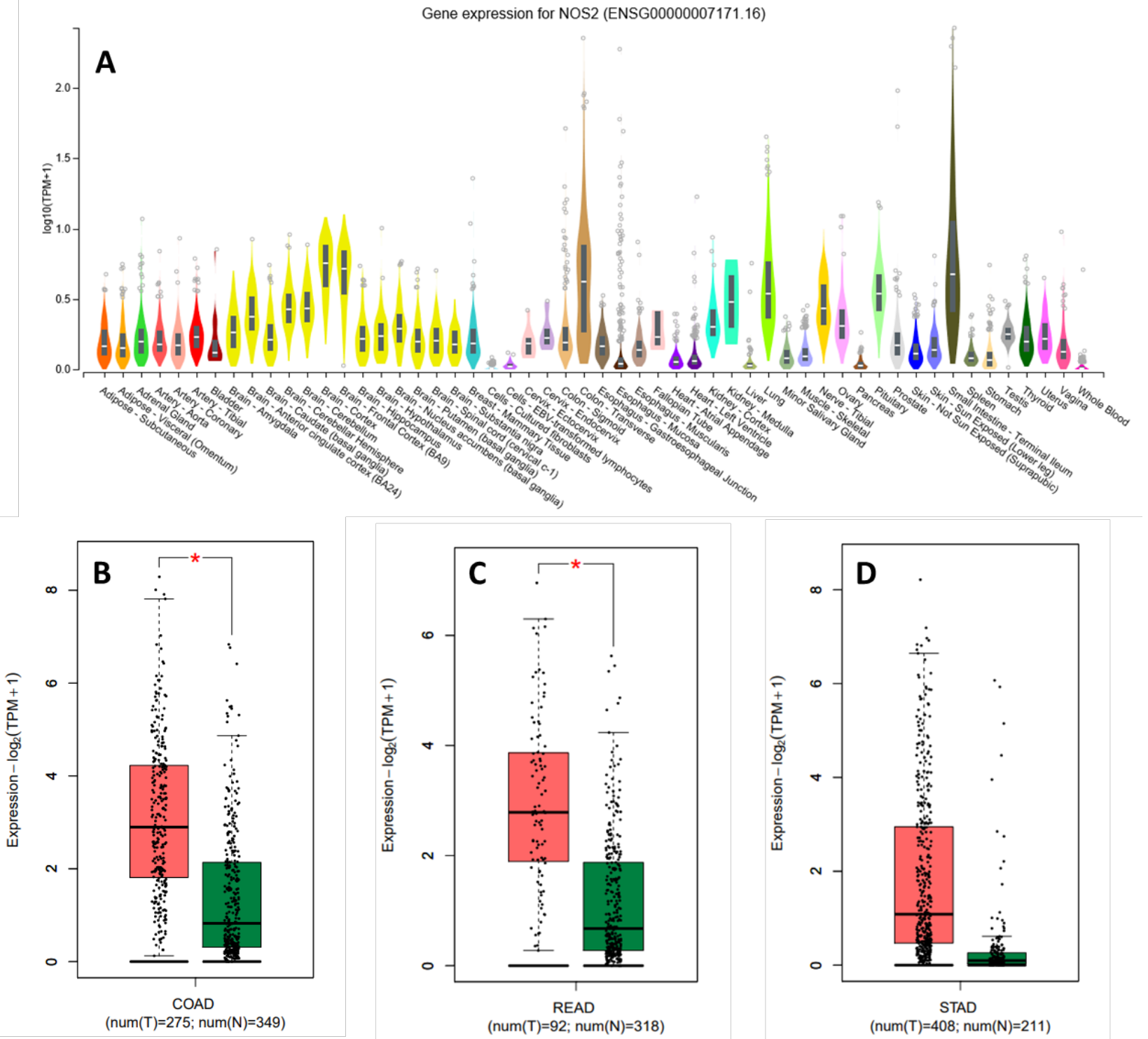

Figure 1: iNOS/NOS2 mRNA relative expression analysis (A: iNOS/NOS2 gene mRNA relative expression in human body tissues; B: iNOS/ NOS2 gene mRNA relative expression between tumor tissue and normal tissue of colon cancer; C: iNOS/NOS2 gene mRNA relative expression between tumor tissue and normal tissue of rectal cancer. D: iNOS/NOS2 gene mRNA relative expression between cancer tissue and normal tissue of gastric cancer).

substitutions accounted for $57.69 \%, 15.38 \%, 2.56 \%$ of mutations respectively inr colorectal cancer (Fig 2A) and $37.7 \%, 19.6 \%, 0.0 \%$ respectively in gastric cancer. (Fig 2C). The top 3 single nucleotide mutation of iNOS/NOS2 gene were $\mathrm{G}>\mathrm{A}(50.85 \%), \mathrm{C}>\mathrm{T}(20.34 \%), \mathrm{C}>\mathrm{A}(6.78 \%)$ for colorectal cancer (Fig 2B) and $\mathrm{G}>\mathrm{A}(59.38 \%), \mathrm{C}>\mathrm{T}(20.34 \%)$, $\mathrm{T}>\mathrm{C}(12.50 \%)$ for gastric cancer respectively (Fig 2D). The iNOS/NOS2 mutation type, site and frequency in pancancer are demonstrated in Fig2E and Fig 3. Missense mutations were the most common mutation and were found across the whole gene.

\section{Co-expression analysis of iNOS/NOS2 and relevant genes}

The positive and negative co-expressed genes were identified using the LinkedOmics online statistical analysis tool with data form the TCGA database.. The heatmaps of positive and negative co-expressed genes with iNOS/NOS2 for colorectal cancer are showed in Fig 4 A, B and for gastric cancer were demonstrated in Figure $4 \mathrm{C}$, D. The top positive and negative co-expressed genes that correlate with iNOS/NOS2 expression are 


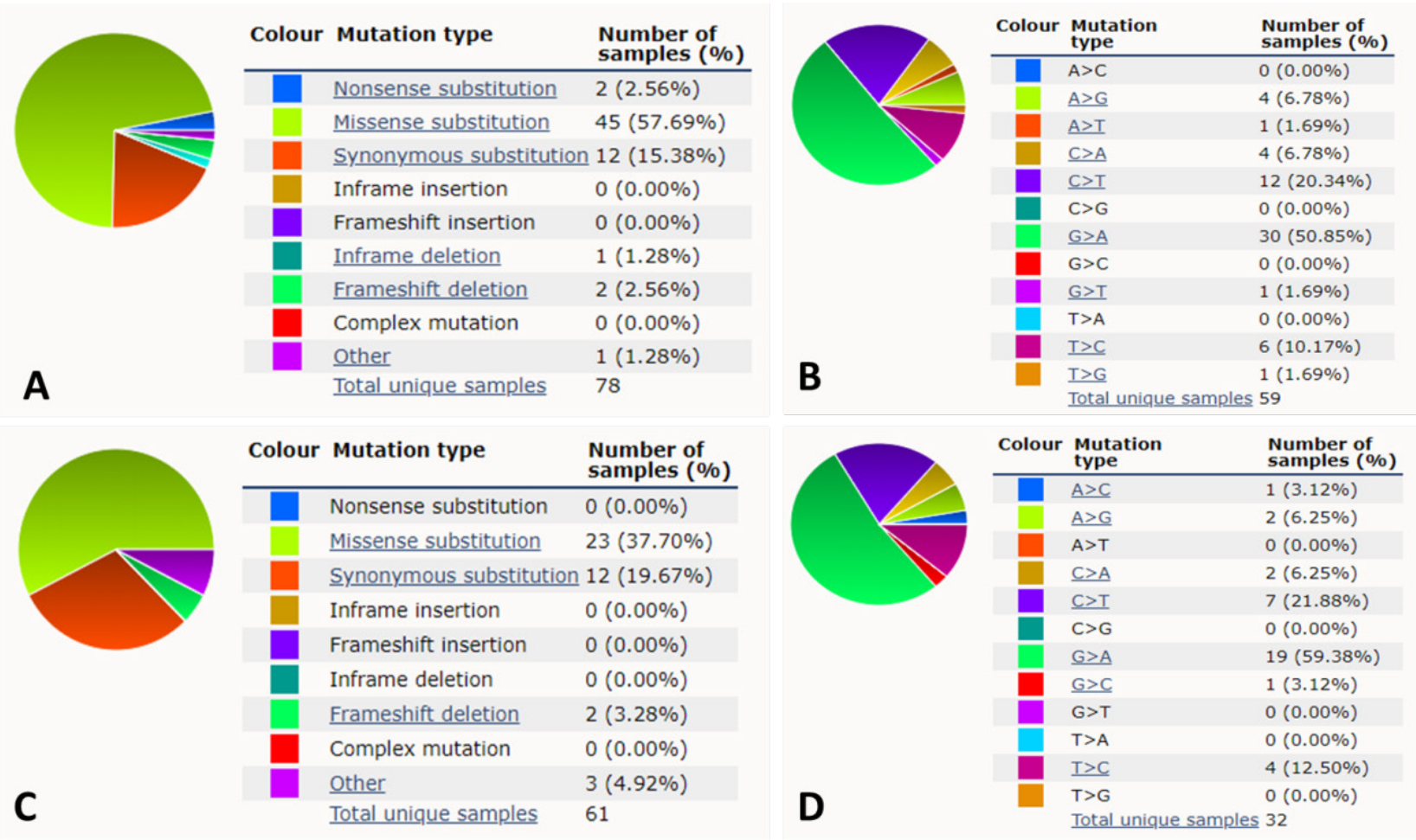

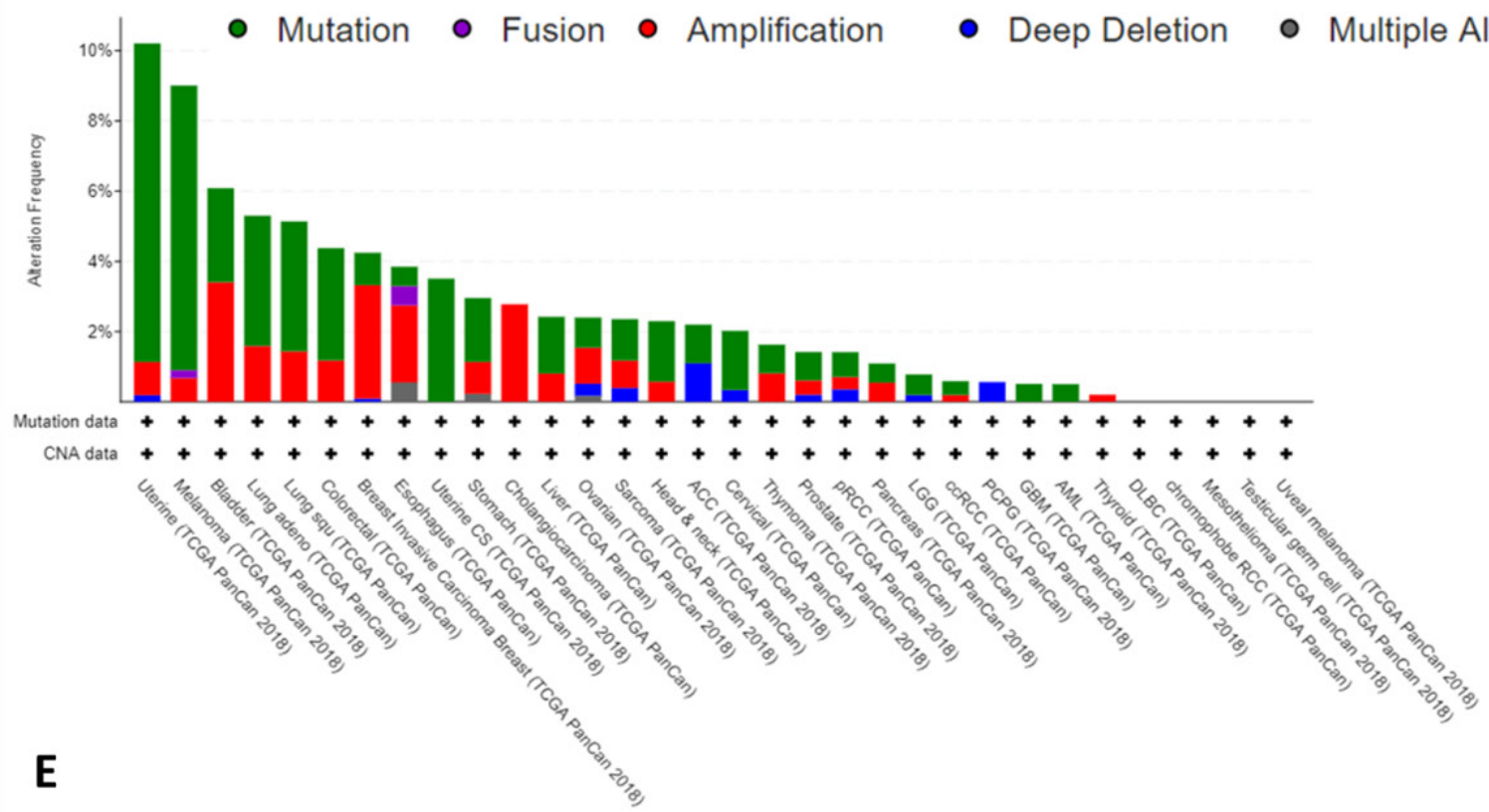

Figure 2: iNOS/NOS2 gene mutation analysis(A: Pie plot of the iNOS/NOS2 mutation frequency of colorectal cancer; B: Pie plot of the iNOS/ NOS2 single nucleotide mutation of colorectal cancer; C: Pie plot of the iNOS/NOS2 mutation frequency of gastric cancer; D: Pie plot of the iNOS/NOS2 single nucleotide mutation of gastric cancer; E:iNOS/NOS2 gene mutation frequency distribution analysis based on TCGA database) The mutation percentage was calculated by the formula of number of cases with mutation/number of cases studied. 


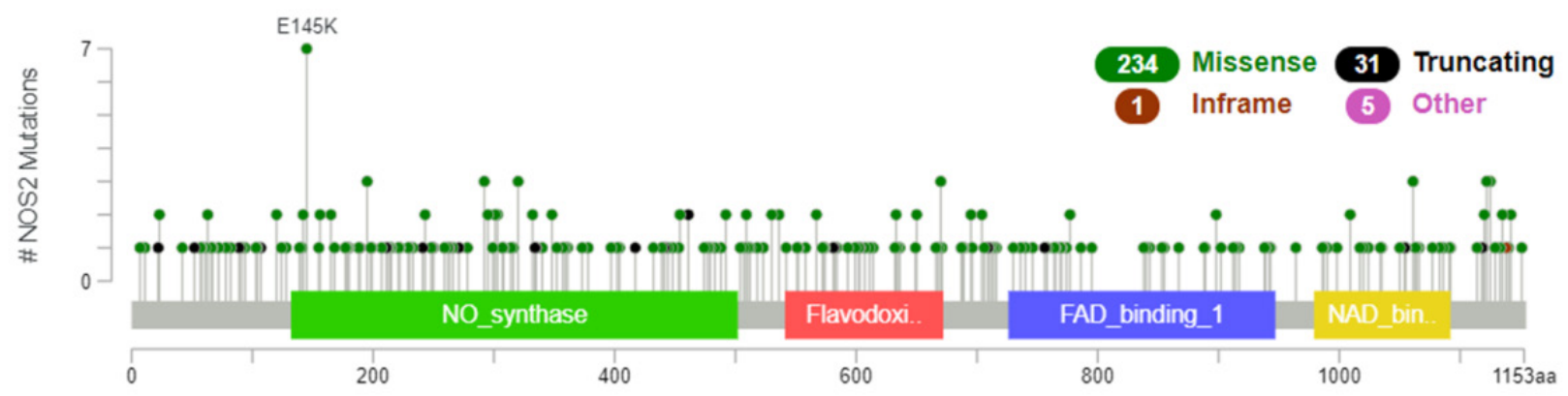

Figure 3: iNOS/NOS2 gene mutation type and location analysis.
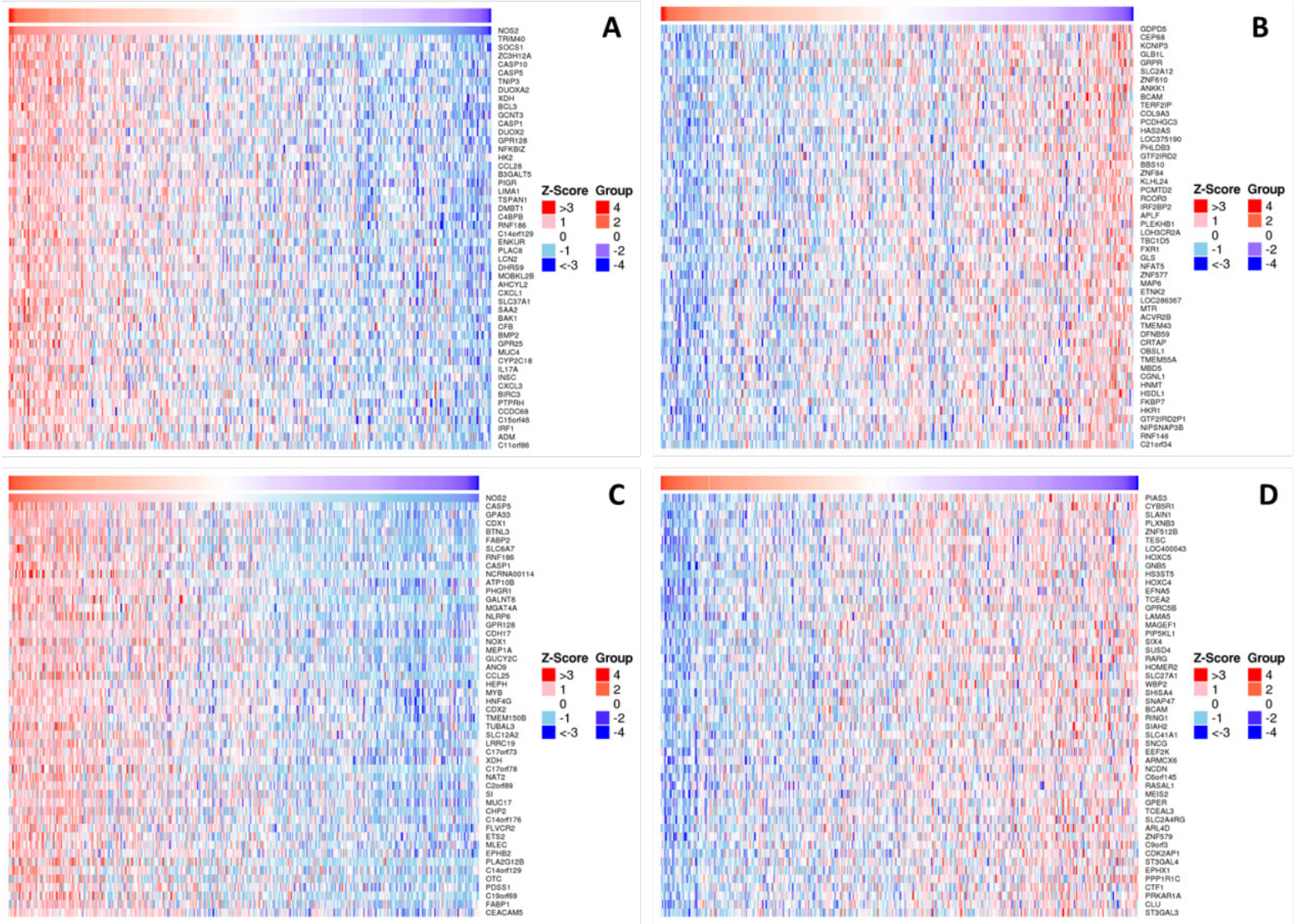

Figure 4: Heatmap of iNOS/NOS2 and co-expressed genes(A: Positive co-expressed genes in colorectal cancer; B: negative co-expressed genes in colorectal cancer; C:positive expressed genes in gastric cancer; D: negative co-expressed genes in gastric cancer). 

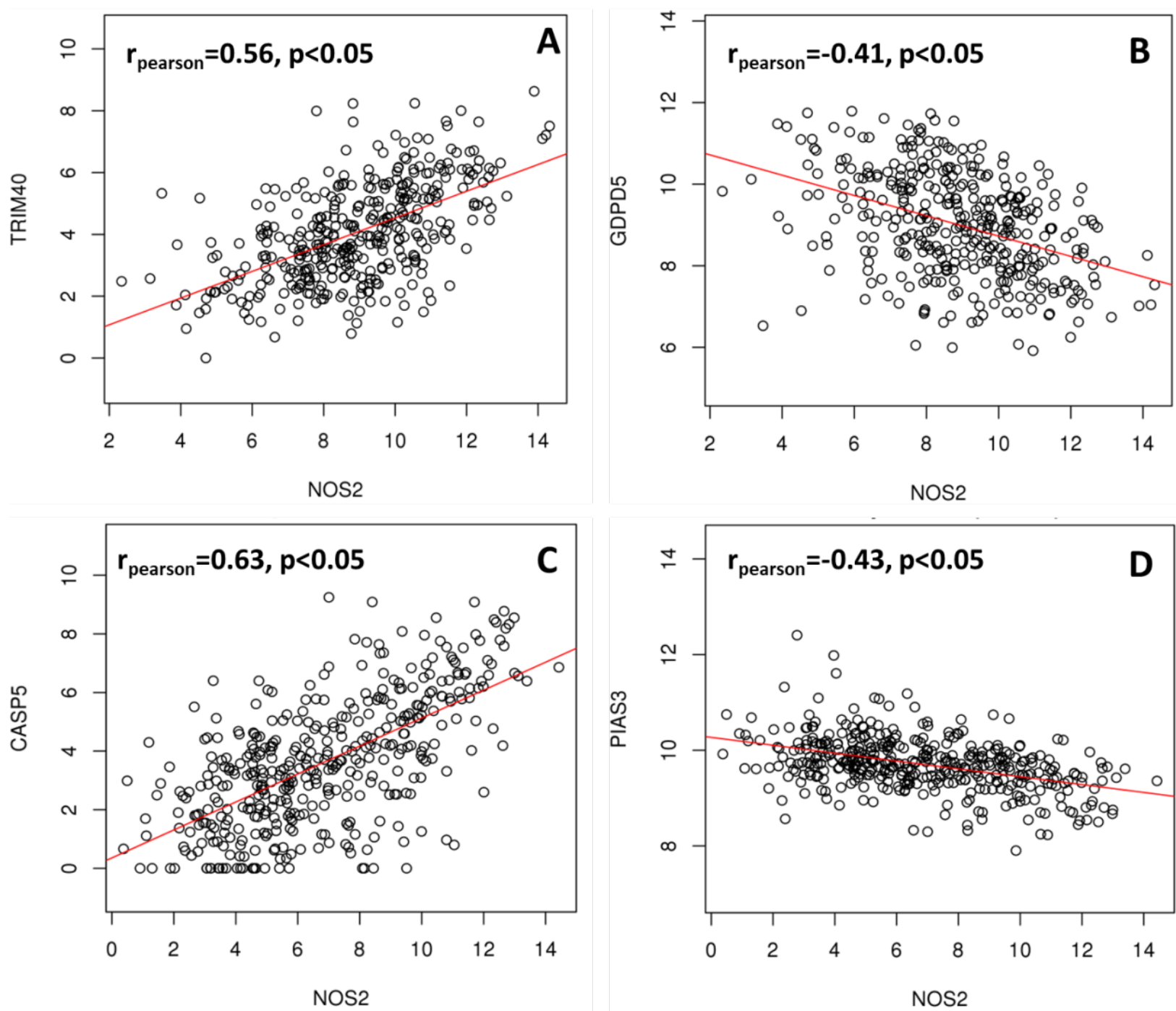

Figure 5: Pearson correlation analysis of the top positive and negative correlated genes with iNOS/NOS2(A: positive correlated genes with iNOS/NOS2 in colorectal cancer; B: negative correlated genes with iNOS/NOS2 in colorectal cancer; C: positive correlated genes with iNOS/ NOS2 in gastric cancer; D: negative correlated genes with iNOS/NOS2 in gastric cancer).

TRIM40 $\left(\mathrm{r}_{\text {pearson }}=0.56, \quad \mathrm{p}<0.05\right)$ and $\operatorname{GDPD} 5\left(\mathrm{r}_{\text {pearson }}=-\right.$ $0.41, \mathrm{p}<0.05)$ respectively in colorectal cancer, (Fig 5) A,B. For gastric cancer, it was $\operatorname{CASP} 5\left(\mathrm{r}_{\text {pearson }}=0.63, \mathrm{p}<0.05\right)$ and $\operatorname{PIAS} 3\left(\mathrm{r}_{\text {pearson }}=-0.43, \mathrm{p}<0.05\right)$, Fig 5 C,D.

\section{PPI network construction}

Twenty one proteins were included in the PPI network. There were 51 nodes and 345 edges with average node degree of 13.5 in the PPI network which indicated the PPI enrichment was significant ( $\mathrm{p}=1.0 \mathrm{e}-16)$, Fig 6.

\section{GO and KEGG pathway analysis}

For gene ontology (GO) analysis, genes that were involved in the PPI network were mainly enriched in response to chemical, response to organic stimulus and nitrogen compound transport in in the aspect of biological process (BP), Fig 7A. For cellular component(CC), the genes were enriched in intracellular organelle and cytoplasm, Fig 7B. For molecular function (MF), the genes were mainly enriched in protein binding and enzyme binding protein dimerization activity, Fig 7C. The KEGG pathway of the included genes were mainly enriched in metabolic pathway, Jak-STAT signaling pathway and pathway in cancer, Table 1 and Fig 7D. 


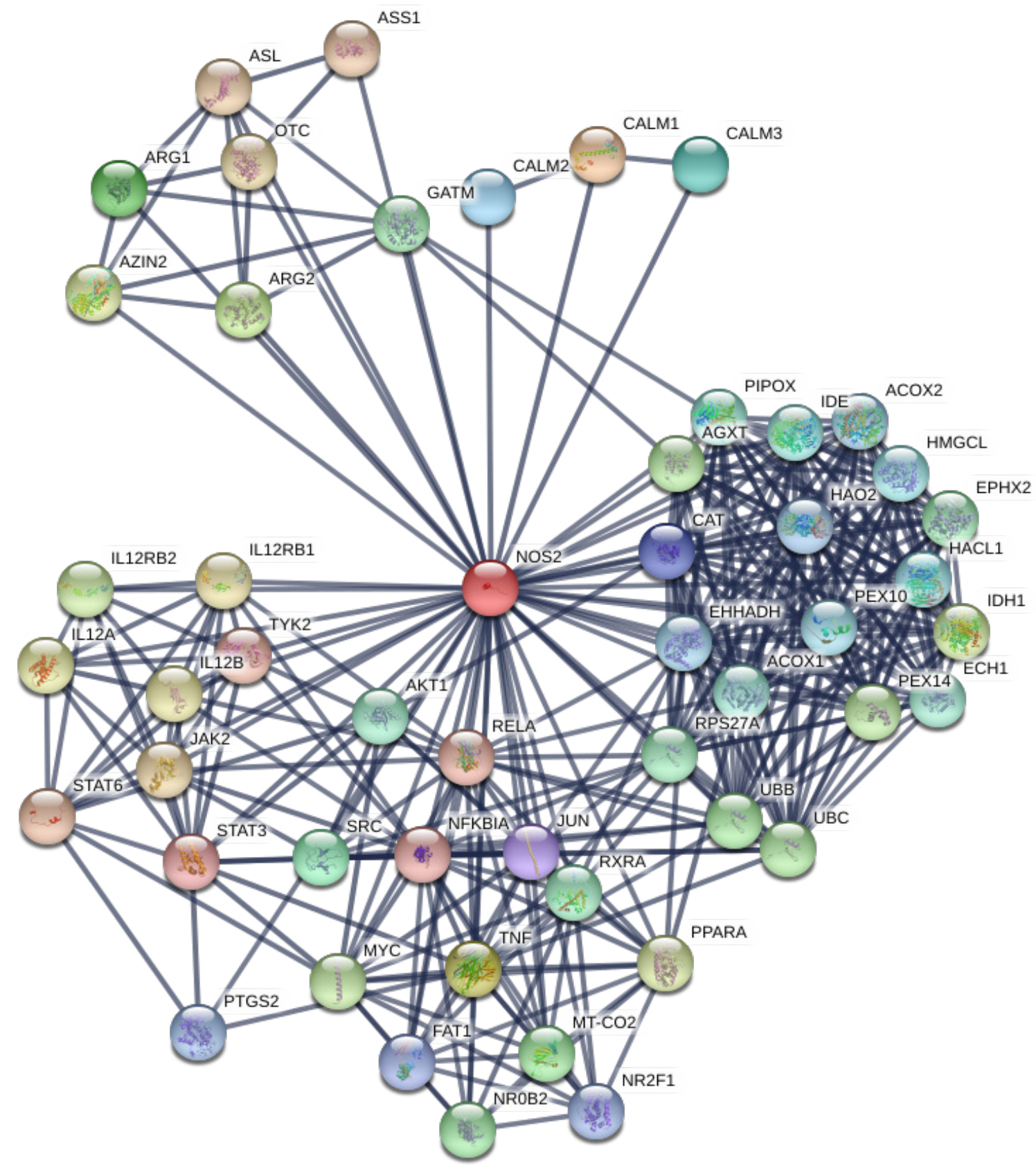

Figure 6: The PPI network of iNOS/NOS2 and relevant gens.

\section{iNOS/NOS2 expression and patients' prognosis}

The disease free survival (DFS) was statistically different between the iNOS/NOS2 high and low expressing groups $(\mathrm{HR}=0.37, \mathrm{p}=0.044)$ in rectal cancer. However, the difference was not statistically significant between iNOS/NOS2 high and low expressing groups for overall survival (OS) or DFS in the colon cancer or gastric cancer ( $p>0.05)$, Fig 8.

\section{Discussion}

In the present work, we systematically analysed the iNOS/NOS2 gene taking into consideration its levels of expression, mutational state, protein-protein interaction and impact on prognosis in colorectal and gastric cancer. We found that iNOS/NOS2 mRNA was up-regulated in both colorectal and gastric cancers, which may indicated that iNOS/NOS2 perform an important role in colorectal 

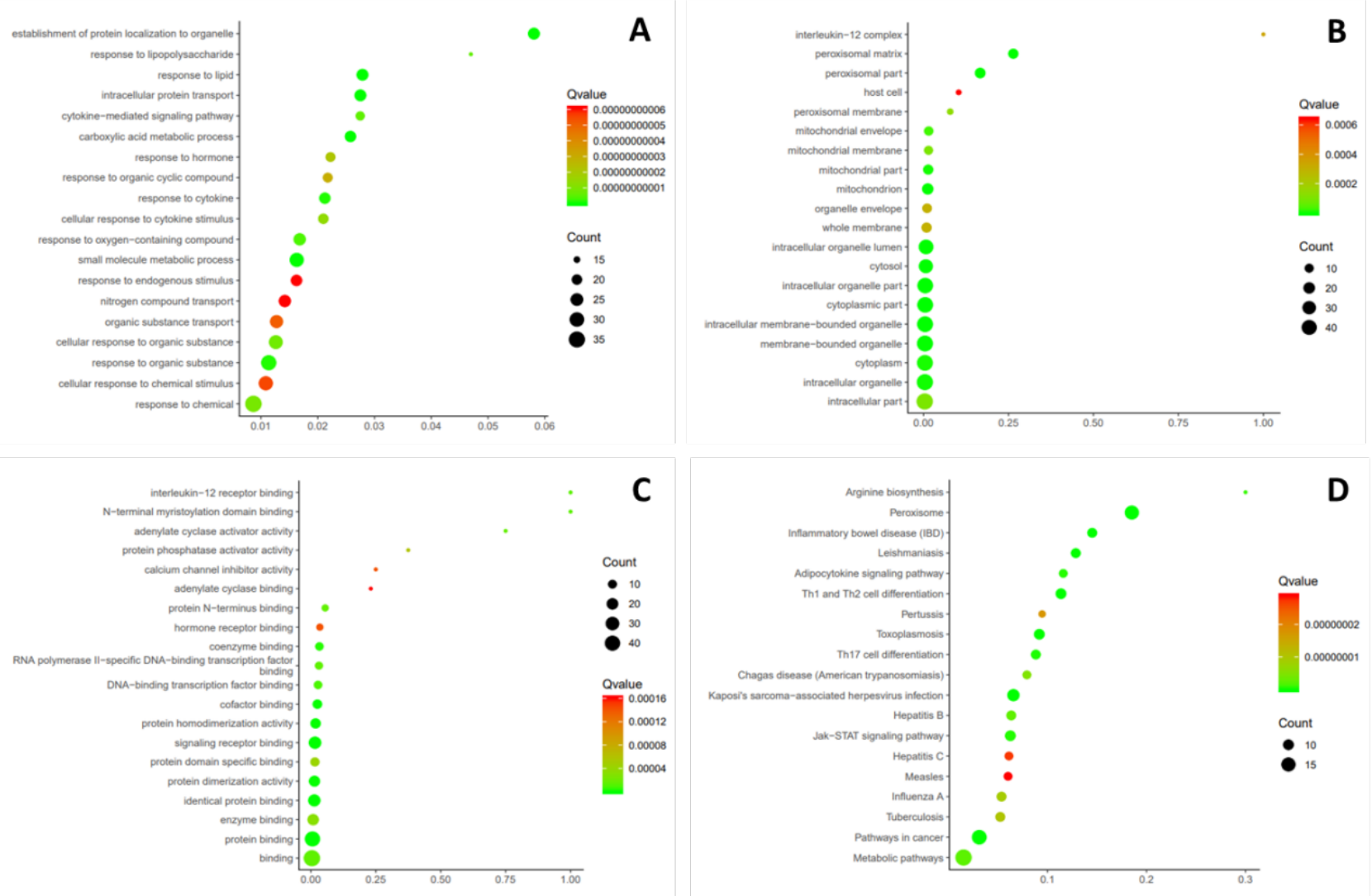

Figure 7: Plot of GO and KEGG pathway enrichment for iNOS/NOS2 and relevant genes (A: biological process enrichment; B:Cellular component enrichment; C:Molecular function enrichment; D:KEGG pathway enrichment).
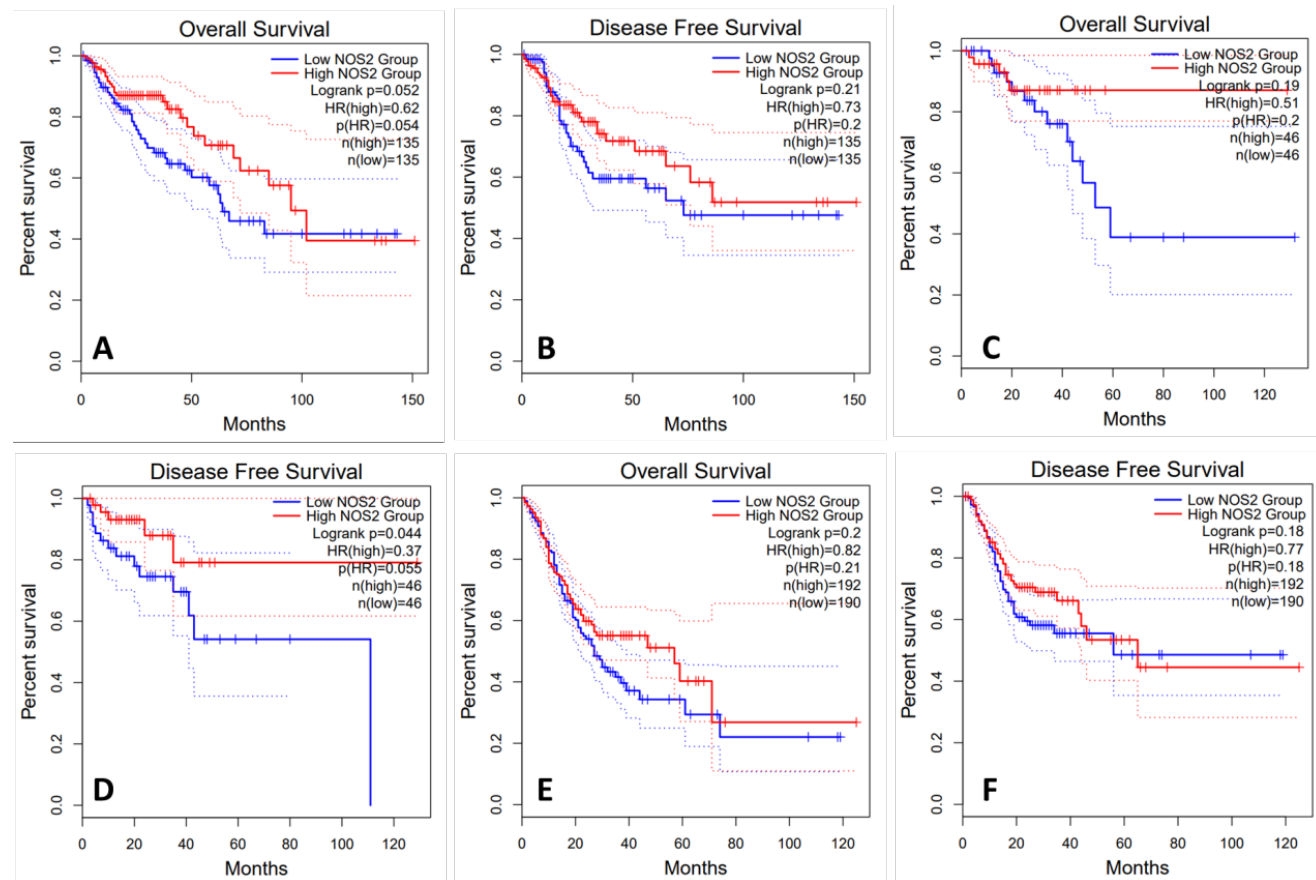

Figure 8: Survival curve between iNOS/NOS2 high and low expression groups (A:OS in colon cancer; B: DFS in colon cancer; C: OS in rectal cancer; D: DFS in rectal cancer; E: OS in gastric cancer; F: DFS in gastric cancer). 
Table 1: The enrichment of the KEGG pathway.

\begin{tabular}{|c|c|c|c|}
\hline Description & Count & Qvalue & GeneRatio \\
\hline Peroxisome & 15 & $3.71 \mathrm{E}-21$ & 0.185185 \\
\hline Th1 and Th2 cell differentiation & 10 & $6.23 \mathrm{E}-12$ & 0.113636 \\
\hline $\begin{array}{l}\text { Kaposi's sarcoma-associated } \\
\text { herpesvirus infection }\end{array}$ & 12 & $6.23 \mathrm{E}-12$ & 0.065574 \\
\hline Pathways in cancer & 16 & $8.11 \mathrm{E}-12$ & 0.031068 \\
\hline $\begin{array}{l}\text { Inflammatory bowel disease } \\
\text { (IBD) }\end{array}$ & 9 & $8.11 \mathrm{E}-12$ & 0.145161 \\
\hline Leishmaniasis & 9 & $1.47 \mathrm{E}-11$ & 0.128571 \\
\hline Toxoplasmosis & 10 & $1.47 \mathrm{E}-11$ & 0.091743 \\
\hline Th17 cell differentiation & 9 & $2.55 \mathrm{E}-10$ & 0.088235 \\
\hline Jak-STAT signaling pathway & 10 & $3.65 \mathrm{E}-10$ & 0.0625 \\
\hline $\begin{array}{l}\text { Adipocytokine signaling } \\
\text { pathway }\end{array}$ & 8 & 4.12E-10 & 0.115942 \\
\hline Arginine biosynthesis & 6 & $8.75 \mathrm{E}-10$ & 0.3 \\
\hline Metabolic pathways & 19 & $2.35 \mathrm{E}-09$ & 0.0152 \\
\hline Hepatitis B & 9 & $2.53 \mathrm{E}-09$ & 0.06338 \\
\hline $\begin{array}{l}\text { Chagas disease (American } \\
\text { trypanosomiasis) }\end{array}$ & 8 & 4.99E-09 & 0.079208 \\
\hline Influenza A & 9 & $9.04 \mathrm{E}-09$ & 0.053571 \\
\hline Tuberculosis & 9 & $1.03 \mathrm{E}-08$ & 0.052326 \\
\hline Pertussis & 7 & $1.76 \mathrm{E}-08$ & 0.094595 \\
\hline Hepatitis C & 8 & $2.71 \mathrm{E}-08$ & 0.061069 \\
\hline Measles & 8 & $2.88 \mathrm{E}-08$ & 0.06015 \\
\hline
\end{tabular}

and gastric tumor carcinogenesis. Gene mutation analysis showed that missense substitutions and synonymous substitutions were the top two most frequent mutation types for colorectal and gastric cancer. The KEGG pathway analysis indicated that iNOS/NOS2 and correlated genes were mainly enriched in Jak-STAT signaling pathway and pathway in cancer. We also found that DFS was statistical different between iNOS/NOS2 high and low expressing groups with statistical difference $(\mathrm{HR}=0.37, \mathrm{p}=0.044)$ in rectal cancer which contradicts the notion thatNOS2 activity results in the promotion of cancer growth and metastasis. The reason for the contradiction may due to: (1) The fact the survival analysis was based on bioinformatics analysis which need to be further verification by local data. (2) the up-regulated NOS2 mRNA may not directly correlated with up-regulation at the protein level. These two reasons may lead to the contradictory findings. It has been reported that iNOS is involved in the process of carcinogenesis of gastric mucosa [16, 17]. It is one of the risk factors in the occurrence and development of gastric cancer. Nitric oxide(NO) can directly or indirectly damage DNA or inhibit DNA repair, and can promote post transcriptional nitrosylation to modify mRNA. Sustained levels of high NO produced by iNOS leads to gene mutation and eventually causes normal mucosa carcinogenesis [18]. The carcinogenic effect of $\mathrm{NO}$ is complex. Under the action of superoxide, $\mathrm{NO}$ forms nitrate, nitrite, $\mathrm{N} 2 \mathrm{O} 3$ and other active nitrogen oxides. Both nitrite and $\mathrm{N} 2 \mathrm{O} 3$ have a strong electron affine nitrosylation group, which can directly crosslink and damage DNA chains. NO can also directly oxidize or nitrogenate caspases or reduce the activity of caspases by inhibiting the release of cytochrome $\mathrm{C}$, thus inhibiting apoptosis of tumor cells and promote tumor growth [19]. However, high levels of iNOS alone is not sufficient to increase the production of NO due to the tight regulation of iNOS by $\mathrm{BH} 4$. $\mathrm{BH} 4$, also known as sapropterin, is a co-factor of the three aromatic amino acid hydroxylase enzymes, used in the degradation of amino acid phenylalanine and in the biosynthesis of the neurotransmitters serotonin (5-hydroxytryptamine, 5-HT), melatonin, dopamine, norepinephrine (noradrenaline), epinephrine (adrenaline), and is a co-factor for the production of nitric oxide (NO) by the nitric oxide syntheses. Recent studies have shown that BH4 plays a role in the occurrence and development of malignant tumor [20, 21]. However, BH4 can also inhibit tumor growth by enhancing the activity of cytotoxic T cells. BH4 had a dual nature both inhibiting and promoting cancer, which suggested that the role of $\mathrm{BH} 4$ in the occurrence and development of malignant tumors still needs to be explored further. More experimental studies on the metabolic mechanism of BH4 are urgently needed, and targeting BH4 biosynthesis pathway are expected to be new methods for the treatment of malignant tumors. Interestingly, the brother compound of $\mathrm{BH} 4$ namely neopterin has been demonstrated to be a strong predictor of outcome in various tumor diseases including colorectal cancer [22], with high concentrations of neopterin being associated with reduced survival [23]. Brandacher and his colleges compared the effect of four-amino tetrahydrobiopterin with that of the unsubstituted parent compound tetrahydrobiopterin and of N6-(iminoethyl)L-lysine (L-NIL), a nonpterin inhibitor of inducible nitric oxide synthase using a murine cardiac transplant model. They found that allograft survival was significantly prolonged by tetrahydrobiopterin and cyclosporin A, but not by L-NIL although decreased plasma nitrite and nitrate levels confirmed nitric oxide synthase inhibition in vivo. This results indicated that the four-amino 
substitution is not required for the immunosuppressive effect of tetrahydrobiopterin compounds [23]. Studies have shown that hypoxia up-regulates the expression of iNOS [24]. Nitric oxide can induce mitosis in endothelial cells, induce the expression of vascular endothelial growth factor(VEGF) in cancer cells and formation of new blood vessels in tumor tissues, thereafter promoting tumor metastasis, tumor growth and metastasis [25].

In the 1980s, NO was firstly recognized as a bioactive molecule in human body. There are two sources of NO in human body: non-enzymatic and enzymatic. Endogenous NO is produced by a multi-step redox reaction between L-arginine (LArg) and oxygen molecules catalyzed by nitric oxide synthase (NOS) [26, 27]. NO has dual effects on tumor growth $[28,29]$. Its tumor promoting effect is mainly related to DNA damage and inhibition of DNA repair enzyme [30-32], promoting of wild-type tp53 gene mutation [30-33], promoting VEGF expression and inhibiting Caspase activity. Several studies have demonstrated that iNOS can be highly expressed in most tumor tissues, which can increase the permeability of blood vessels, accelerate the formation of tumor blood vessels, and promote the growth and metastasis of tumors $[34,35]$.

The high concentration of NO produced by iNOS can participate in the development of inflammation and tumor formation through the following effects [36-39]: (1) producing carcinogenic nitrosamines, inducing DNA base deamination and gene mutation; (2) excessive NO can cause cytotoxicity of membrane lipid peroxidation; (3) NO plays an important role in the proliferation and migration of vascular endothelial cells promoted by VEGF. In addition, excessive iNOS can also inhibit the apoptosis of tumor cells. Because the half-life of NO in vivo is extremely short, the production of NO can be indirectly reflected by detecting iNOS. It is also believed that iNOS may be related to the imbalance of cell proliferation/apoptosis, tumor neovascularization, inhibition of immune function on tumor cells. However the exact mechanism of iNOS involved in tumorigenesis and development is still unclear.

\section{Conclusion}

iNOS/NOS2 mRNA was up-regulated in tumor tissue compared to corresponding normal tissue in colorectal and gastric cancer which may involve in the colorectal and gastric development. However, our work has limitations. Firstly, this work was performed based on bioinformatics analysis which need further confirmation by own data. Second, the molecular mechanism of iNOS/
NOS2 involved in the colorectal and gastric cancer was not clearly addressed. Cell experiments are needed to further illustrate it molecular function in colorectal and gastric cancer development.

Data Availability Statement: The datasets generated during and analysed during the current study are available from the corresponding author on reasonable request.

Conflict of interest: Authors state no conflict of interest

\section{References}

1. Siegel RL, Miller KD, Jemal A. Cancer statistics, 2020. CA Cancer J Clin. 2020 Jan;70(1):7-30.

2. Takii Y, Maruyama S, Nogami H. Can the prognosis of colorectal cancer be improved by surgery? World J Gastrointest Surg. 2016 Aug;8(8):574-7.

3. Li J. Gastric Cancer in Young Adults: A Different Clinical Entity from Carcinogenesis to Prognosis. Gastroenterol Res Pract. 2020 Mar;2020:9512707.

4. Sawaki K, Kanda M, Kodera Y. Review of recent efforts to discover biomarkers for early detection, monitoring, prognosis, and prediction of treatment responses of patients with gastric cancer. Expert Rev Gastroenterol Hepatol. 2018 Jul;12(7):65770.

5. Johnson LA, June $\mathrm{CH}$. Driving gene-engineered $\mathrm{T}$ cell immunotherapy of cancer. Cell Res. 2017 Jan;27(1):38-58.

6. Chiang YT, Wang K, Fazli L, Qi RZ, Gleave ME, Collins CC, et al. GATA2 as a potential metastasis-driving gene in prostate cancer. Oncotarget. 2014 Jan;5(2):451-61.

7. Fu HC, Chuang IC, Yang YC, Chuang PC, Lin H, Ou YC, et al. Low P16 ${ }^{\text {INK4A }}$ Expression Associated with High Expression of Cancer Stem Cell Markers Predicts Poor Prognosis in Cervical Cancer after Radiotherapy. Int J Mol Sci. 2018 Aug;19(9):19.

8. Macedo J, Silva E, Nogueira L, Coelho R, da Silva J, Dos Santos A, et al. Genomic profiling reveals the pivotal role of hrHPV driving copy number and gene expression alterations, including mRNA downregulation of TP53 and RB1 in penile cancer. Mol Carcinog. 2020 Jun;59(6):604-17.

9. Aaltoma SH, Lipponen PK, Kosma VM. Inducible nitric oxide synthase (iNOS) expression and its prognostic value in prostate cancer. Anticancer Res. 2001 Jul-Aug;21 4B:3101-6.

10. Jabłońska E, Puzewska W, Marcińczyk M, Grabowska Z, Jabłoński J. iNOS expression and NO production by neutrophils in cancer patients. Arch Immunol Ther Exp (Warsz). 2005 MarApr;53(2):175-9.

11. Barani R, Motalleb G, Maghsoudi H. Evaluation of iNOS Expression in Esophageal Cancer Patients. Gastrointest Tumors. 2016 Sep;3(1):44-58.

12. Li QT, Huang ZZ, Chen YB, Yao HY, Ke ZH, He XX, et al. Integrative Analysis of Siglec-15 mRNA in Human Cancers Based on Data Mining. J Cancer. 2020 Feb;11(9):2453-64.

13. Vasaikar SV, Straub P, Wang J, Zhang B. LinkedOmics: analyzing multi-omics data within and across 32 cancer types. Nucleic Acids Res. 2018 Jan;46 D1:D956-63. 
14. Szklarczyk D, Gable AL, Lyon D, Junge A, Wyder S, Huerta-Cepas J, et al. STRING v11: protein-protein association networks with increased coverage, supporting functional discovery in genome-wide experimental datasets. Nucleic Acids Res. 2019 Jan;47 D1:D607-13.

15. Fortino V, Alenius H, Greco D. BACA: bubble chArt to compare annotations. BMC Bioinformatics. 2015 Feb;16(1):37.

16. Wang GY, Ji B, Wang X, Gu JH. Anti-cancer effect of iNOS inhibitor and its correlation with angiogenesis in gastric cancer. World J Gastroenterol. 2005 Jul;11(25):3830-3.

17. Song ZJ, Gong P, Wu YE. Relationship between the expression of iNOS,VEGF,tumor angiogenesis and gastric cancer. World J Gastroenterol. 2002 Aug;8(4):591-5.

18. Rao CV. Nitric oxide signaling in colon cancer chemoprevention. Mutat Res. 2004 Nov;555(1-2):107-19.

19. Padgett CM, Whorton AR. Cellular responses to nitric oxide: role of protein S-thiolation/dethiolation. Arch Biochem Biophys. 1998 Oct;358(2):232-42.

20. Ziche M, Morbidelli L. Molecular regulation of tumour angiogenesis by nitric oxide. Eur Cytokine Netw. 2009 Dec;20(4):164-70.

21. Chen L, Zeng X, Wang J, Briggs SS, O'Neill E, Li J, et al. Roles of tetrahydrobiopterin in promoting tumor angiogenesis. Am J Pathol. 2010 Nov;177(5):2671-80.

22. Schroecksnadel S, Sucher R, Kurz K, Fuchs D, Brandacher $\mathrm{G}$. Influence of immunosuppressive agents on tryptophan degradation and neopterin production in human peripheral blood mononuclear cells. Transpl Immunol. 2011 Sep;25(23):119-23.

23. Brandacher G, Maglione M, Schneeberger S, Obrist P, Thoeni $\mathrm{G}$, Wrulich OA, et al. Tetrahydrobiopterin compounds prolong allograft survival independently of their effect on nitric oxide synthase activity. Transplantation. 2006 Feb;81(4):583-9.

24. Guo G, Bhat NR. Hypoxia/reoxygenation differentially modulates NF-kappaB activation and iNOS expression in astrocytes and microglia. Antioxid Redox Signal. 2006 MayJun;8(5-6):911-8.

25. Akhavan-Sigari R, Gaab MR, Rohde V, Brandis A, Tezval H, Abili $M$, et al. Expression of vascular endothelial growth factor receptor 2 (VEGFR-2), inducible nitric oxide synthase (iNOS), and Ki-M1P in skull base chordoma: a series of 145 tumors. Neurosurg Rev. 2014 Jan;37(1):79-88.

26. MacKenzie A, Wadsworth RM. Extracellular L-arginine is required for optimal NO synthesis by eNOS and iNOS in the rat mesenteric artery wall. Br J Pharmacol. 2003 Aug;139(8):148797.

27. Briones AM, Alonso MJ, Marín J, Salaices M. Role of iNOS in the vasodilator responses induced by L-arginine in the middle cerebral artery from normotensive and hypertensive rats. $\mathrm{Br}$ J Pharmacol. 1999 Jan;126(1):111-20.

28. Sanhueza C, Araos J, Naranjo L, Barros E, Subiabre M, Toledo F, et al. Nitric oxide and $\mathrm{pH}$ modulation in gynaecological cancer. J Cell Mol Med. 2016 Dec;20(12):2223-30.

29. Huang Z, Fu J, Zhang Y. Nitric Oxide Donor-Based Cancer Therapy: advances and Prospects. J Med Chem. 2017 Sep;60(18):7617-35.

30. Oleson BJ, Corbett JA. Dual Role of Nitric Oxide in Regulating the Response of $\beta$ Cells to DNA Damage. Antioxid Redox Signal. 2018 Nov;29(14):1432-45.
31. Tamir S, Burney S, Tannenbaum SR. DNA damage by nitric oxide. Chem Res Toxicol. 1996 Jul-Aug;9(5):821-7.

32. Keefer LK, Wink DA. DNA damage and nitric oxide. Adv Exp Med Biol. 1996;387:177-85.

33. Ambs S, Hussain SP, Harris CC. Interactive effects of nitric oxide and the $\mathrm{p} 53$ tumor suppressor gene in carcinogenesis and tumor progression. FASEB J. 1997 May;11(6):443-8.

34. Anadol E, Yar Saglam AS, Gultiken N, Karakas K, Alcigir E, Alkan $\mathrm{H}$, et al. Expression of iNOS, COX-2 and VEGF in canine mammary tumours and non-neoplastic mammary glands: association with clinicopathological features and tumour grade. Acta Vet Hung. 2017 Sep;65(3):382-93.

35. Sappayatosok K, Maneerat Y, Swasdison S, Viriyavejakul P, Dhanuthai K, Zwang J, et al. Expression of pro-inflammatory protein, iNOS, VEGF and COX-2 in oral squamous cell carcinoma (OSCC), relationship with angiogenesis and their clinicopathological correlation. Med Oral Patol Oral Cir Bucal. 2009 Jul;14(7):E319-24.

36. Huerta S, Chilka S, Bonavida B. Nitric oxide donors: novel cancer therapeutics (review) [review]. Int J Oncol. 2008 Nov;33(5):909-27.

37. Wink DA, Ridnour LA, Hussain SP, Harris CC. The reemergence of nitric oxide and cancer. Nitric Oxide. 2008 Sep;19(2):65-7.

38. Moochhala S, Rajnakova A. Role of nitric oxide in cancer biology. Free Radic Res. 1999 Dec;31(6):671-9.

39. Xu W, Liu LZ, Loizidou M, Ahmed M, Charles IG. The role of nitric oxide in cancer. Cell Res. 2002 Dec;12(5-6):311-20. 